\title{
The comorbidity of headaches in pediatric epilepsy patients: How common and what types?
}

Hanin Al-Gethami, MD, Muhammad TalalAlrifai, MD, Ahmed AlRumayyan, MD, Waleed AlTuwaijri, MD, Duaa Baarmah, MD.

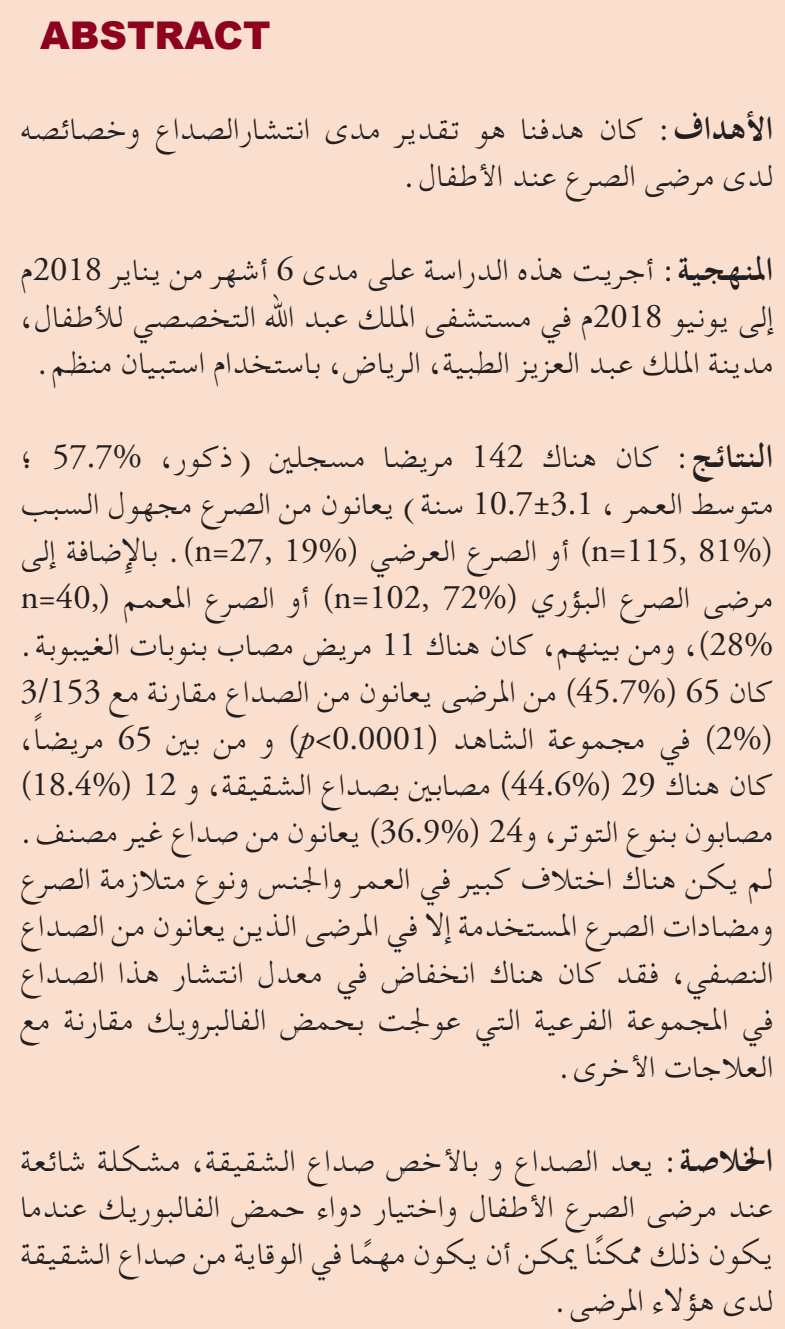

Objectives: To estimate the prevalence and characteristics of headache in pediatric epileptic patients.

Methods: This cross-sectional study was performed over 6 months period from January 2018 to June 2018 at King Abdullah Specialist Children Hospital, King Abdulaziz Medical City, Riyadh, Kingdom of Saudi Arabia using a structured questionnaire in pediatric patients with epilepsy.

Results: There were 142 patients enrolled (males, $57.7 \%$; average age, $10.7 \pm 3.1$ years) with idiopathic epilepsy $(\mathrm{n}=115,81 \%)$ or symptomatic epilepsy $(\mathrm{n}=27,19 \%)$. Additionally, patients had focal epilepsy $(n=102,72 \%)$ or generalized epilepsy $(n=40,28 \%)$, and among them, 11 had absence epilepsy. Overall, 65 (45.7\%) patients had headaches compared with $3 / 153$ $(2 \%)$ in the control group $(p<0.0001)$. Among the 65 patients with headaches, $29(44.6 \%)$ had migrainetype, $12(18.4 \%)$ had tension-type, and $24(36.9 \%)$ had unclassified headache. There was no significant difference in age, gender, type of epilepsy syndrome, and antiepileptic used except in patients with or without headache. For migraine patients, there was a lower headache prevalence in the subgroup treated with valproic acid compared with other treatments.

Conclusion: Headache, predominantly migraine, is a common problem in pediatric epileptic patients and choosing valproic acid when possible can be important in preventing migraine in these patients.

Neurosciences 2019; Vol. 24 (4): 284-289 doi: 10.17712/nsj.2019.4.20190043

From Neuro-science Center (Al-Gethami), King Fahd Medical City, and from Pediatric Neurology Division (Alrifai, Al-Rumayyan, AlTuwaijri, Ba-armah), Pediatric Department, King Abdullah Children Specialist Hospital, King Abdulaziz Medical City, Riyadh, Kingdom of Saudi Arabia.

Received 15th May 2019. Accepted 31st July 2019.

Address correspondence and reprint request to: Dr. Muhammad Talal Alrifai, Pediatric Neurology Consultant, Pediatric Neurology Division, Pediatric Department, King Abdullah Children Specialist Hospital, King Abdulaziz Medical City, Riyadh, Kingdom of Saudi Arabia. E-mail:rifaim@ngha.med.sa

ORCID ID: https://orcid.org/0000-0002-3939-0793 
E pilepsy and headache are chronic paroxysmal disorders that affect adult and pediatric patients ${ }^{1}$ with episodic manifestations. Headache or (cephalalgia) is defined as a feeling of pain in the region of the head or neck. Primary headaches include migraines, tension-type headache, and cluster headache. Epileptic seizure is a brief episode of signs or symptoms caused by abnormal excessive synchronized neuronal activity. ${ }^{2}$ Epilepsy is defined as a condition where the patient has an enduring tendency to have recurrent unprovoked seizures. ${ }^{2}$ These two disorders coexist in some patients. ${ }^{3}$ There are few studies on the comorbidity of headaches in children with epilepsy. ${ }^{4-6}$ Other studies reported a significant association between migraine and epilepsy. ${ }^{1,7-9}$ Additionally, the genetic predisposition for both entities was reported in some forms of channelopathy, ${ }^{10}$ and others found more prevalence of migraine headache in specific diseases in pediatric like benign epilepsy with centrotemporal spikes and juvenile myoclonic epilepsy. ${ }^{11}$ Seizure-associated headache is common, with an incidence of $42-51 \%$ in adult epileptic patients. ${ }^{12}$ However, for pediatric patients, it is often neglected by parents and physicians because of other neurological manifestations of the seizure such as loss of consciousness and motor components, ${ }^{13}$ and approximately $36 \%$ of the parents were reported to be unaware that their children experienced headache. ${ }^{14} \mathrm{It}$ is our experience that headache is a common problem in up to $50 \%$ of epilepsy patients but we do not know exactly the prevalence, in addition to what type of headache is most commonly found in epileptic pediatric patients. Because of few reports on this topic have conflicting results, the objective of this study was to evaluate the prevalence and characteristics of headache in children with epilepsy who were seen at one center in Saudi Arabia.

Methods. This cross-sectional study was performed over a 6-month period from January 2018 to June 2018 at King Abdullah Specialist Children Hospital, King Abdulaziz Medical City, Riyadh.

Inclusion criteria were as follows: pediatric epilepsy patients (5-16 years of age) and who were mentally normal and could communicate their symptoms with and without other comorbidities like diabetes,

Disclosure. Authors have no conflict of interests, and the work was not supported or funded by any drug company. asthma, or renal disease. Epilepsy syndrome patients were excluded since most of them mentally affected. Patients with intellectual disability and patients with unclear information supplied by the patient or their family were also excluded. We had up to 20 patients / pediatric neurology clinic, and 8 clinic/ week, so 142 patients met our inclusion criteria and more than 20 patients were excluded. Patients' parents/guardians provided informed consent and the study was approved by an ethics board at our hospital. Also, the study was according to principles of Helsinki Declaration.

Patients and their family were interviewed by one of the researchers (H.G.) using a structured questionnaire during their visit to the clinic. The questionnaire was structured to include demographic and clinical information about the symptoms of epilepsy and headache when present. Each patient was asked specifically if had headache, and if yes, the exact age of onset, how many attacks/ month, the relationship to seizure in timing, quality of headache, location, severity, other associated symptoms like nausea, vomiting, photophobia, phono-phobia, facial pain, orbital pain, and eye swelling, if had aura before headache, and frequency of headache. Further data regarding neurological exam, electrophysiological and neuroimaging studies were obtained from the patients' charts.

The control group comprised siblings of the patients with epilepsy who were free from epilepsy and who were 5-16 years of age, otherwise healthy, and could communicate their symptoms.

Headache was diagnosed based on the International Classification of Headache Disorders (ICHD-II) criteria, ${ }^{15}$ and epilepsy was diagnosed based on the International League Against Epilepsy (ILAE) criteria. ${ }^{16}$ Seizures were considered frequent if they occurred 2 or more times per month; otherwise, they are considered to be infrequent. Seizure-associated headache was defined as a headache starting within 1 hour before or after the seizure. Headache was either classified to be peri-ictal or inter-ictal headache, however, the peri-ictal headache can be classified to pre- ictal, ictal, and postictal headache. Pre-ictal headache is a headache that began before the seizure and lasted until its onset. ictal headache defined as epileptic seizures in which headache is one of the constituents of the epileptic seizure, besides other manifestations like sensory-motor, psychiatric or non-autonomic one. A headache that occurred only after the seizure is called a post-ictal headache. This classification is based on the IHS criteria. ${ }^{17-19}$ Other headaches are considered inter-ictal.

The data were entered into a database and analyzed using SAS (Statistical Analysis Software). Statistical 
Table 1 - Demographics and presence of headaches in the patient and control groups.

\begin{tabular}{lccc}
\hline Demographics & $\begin{array}{c}\text { Patients } \\
\mathbf{n}=142\end{array}$ & $\begin{array}{c}\text { Control } \\
\mathbf{n}=153\end{array}$ & $P$-value \\
\hline Gender (male) & $82(57.7 \%)$ & $85(55.5 \%)$ & 0.70 \\
$\begin{array}{l}\text { Age (years; mean, } \\
\text { standard deviation) }\end{array}$ & $10.7( \pm 3.1)$ & $10.6( \pm 2.9)$ & 0.35 \\
Headache & $65(45.7 \%)$ & $3(1.96 \%)$ & $<0.00001$ \\
\hline
\end{tabular}

analysis tests for both continuous and categorical variables were used as appropriate. No other systems were used.

We calculated measures of the association between variables expressed as the $p$-value, and for each test the level of significance was set at 0.05 .

Results. There were 142 patients with epilepsy who fulfilled the entry criteria for this study. Among these patients, 82 were males $(57.7 \%$ ) and the overall average age for both male and female was $10.7 \pm 3.1$ years. Idiopathic epilepsy was present in 115 (81\%) patients while symptomatic epilepsy was present in 27 (19\%) patients. Focal epilepsy was present in $102(72 \%)$ and generalized epilepsy was present in $40(28 \%)$ patients, 11 of whom had absence epilepsy. Neuroimaging (CT or MRI) was performed in all patients, and 30/142 $(21 \%)$ of the studies showed abnormal results, such as focal cortical dysplasia and mild ischemic brain insult.
The EEG which is an electrophysiological monitoring method used to record electrical activity of the brain, was also performed for 141 patients, and 59 (41.8\%) showed normal results, 73 (51.8\%) showed epileptiform activity focal and generalized interictal discharges with majority had generalized discharges, and 9 (6.4\%) showed focal or generalized slowing.

Among the 142 patients, 65 (45.7\%) had headache compared with $3 / 153$ (2\%) of the control group $(p<0.0001)$. The 2 groups were matched for age and gender (Table 1).

Among the 65 patients with headache, 29 (44.6\%) patients had migraine-type headache, 12 (18.4\%) patients had tension-type headache, and 24 (36.9\%) patients had headache that was an unclassified type. The headache and epilepsy started at the same year in 28 patients, and headache preceded epilepsy in total of 7 patients, it was 1 -year period in 5 patients, 2 years period in 1 patient, and 3 years period in 1 patient.

Epilepsy started 1 year before headache in 10 patients, 2 years before in 3 patients, 3 years before in 6 patients, 4 years before in 5 patients; 5 years before in 2 patients; and 6 years before in 2 patients, 8 years before in one patient, and 11 years before in one patient.

For the timing of headache in relation to seizures, 56 out of $65(86 \%)$ patients had interictal headaches, $5(7.6 \%)$ patients had post-ictal headaches, 17 (26\%) patients had preictal headaches, and 3 patients had ictal headache $(4.6 \%)$. In fact, some patients have mixed

Table 2 - Comparison of patients with headache to patients without headache.

\begin{tabular}{lccc}
\hline Characteristics & $\begin{array}{c}\text { Patients with } \\
\text { headache } \mathbf{n}=65\end{array}$ & $\begin{array}{c}\text { Patients without } \\
\text { headache } \mathbf{n}=77\end{array}$ & $P$-value \\
$\mathbf{n}(\%)$ & \\
\hline Gender (male [\%]) & $35(53.8)$ & $47(61)$ & 0.38 \\
Age (years; mean \pm standard deviation) & $11( \pm 3.1)$ & $10.6( \pm 3.2)$ & 0.22 \\
Type of seizures: Partial (\%) & $47(72.3)$ & $55(71.4)$ & 0.90 \\
Generalized (\%) & $18(27.6)$ & $22(28.5)$ & \\
Epilepsy syndrome: Idiopathic & $54(83)$ & $61(79.2)$ & 0.55 \\
Symptomatic & $11(16.9)$ & $16(20.7)$ & \\
Frequent seizures & $24(36.9)$ & $29(37.6)$ & 0.92 \\
Lobe type seizure: Occipital lobe seizure & $7(10.7)$ & $9(11.6)$ & 0.86 \\
Temporal lobe seizure & $19(29.2)$ & $22(28.5)$ & 0.93 \\
Parietal lobe seizure & $3(4.6)$ & $5(6.4)$ & 0.68 \\
Frontal lobe seizure & $17(26)$ & $17(22)$ & 0.57 \\
Drug used: Carbamazepine & $25(38.4)$ & $30(38.9)$ & 0.95 \\
Levetiracetam & $17(26)$ & $18(23.3)$ & 0.70 \\
Valproic acid & $20(30.7)$ & $24(31)$ & 0.95 \\
Topiramate & $3(4.6)$ & $8(10.3)$ & 0.22 \\
Poly-therapy & $14(21.5)$ & $11(14.2)$ & 0.25 \\
Others anti-epileptic drugs & $13(20)$ & $7(9)$ & 0.06 \\
\hline
\end{tabular}


Table 3 - Comparison of patient subgroups with migraine or non-migraine headaches.

\begin{tabular}{lccc}
\hline Patient subgroups & Migraine $\mathbf{n}=29$ & No migraine $\mathbf{n}=36$ & $P$-value \\
& \multicolumn{2}{c}{$\mathbf{n}(\%)$} \\
\hline Age (years; mean, standard deviation) & $10.9(2.8)$ & $11.1(3.4)$ & 0.39 \\
Gender: male & $16(55)$ & $19(52.7)$ & 0.84 \\
female & $13(44.8)$ & $17(47.2)$ & 0.84 \\
Epilepsy syndrome: Idiopathic & $21(72.4)$ & $22(61.1)$ & 0.33 \\
Symptomatic & $6(20.6)$ & $5(13.8)$ & 0.46 \\
Type of seizure: Focal & $22(75.8)$ & $25(69.4)$ & 0.56 \\
Generalized & $7(24)$ & $11(30.5)$ & 0.56 \\
Focal seizure: occipital lobe & $3(10.3)$ & $3(8.3)$ & 0.78 \\
Temporal lobe & $8(27.5)$ & $10(27.7)$ & 0.98 \\
Parietal lobe & $1(3.4)$ & $2(5.5)$ & 0.68 \\
Frontal lobe & $10(34.4)$ & $6(16.6)$ & 0.09 \\
Medication: Carbamazepine & $12(41.3)$ & $13(36.1)$ & 0.66 \\
Levetiracetam & $9(31)$ & $8(22.2)$ & 0.42 \\
Valproic acid & $5(17.2)$ & $15(41.6)$ & 0.03 \\
Topiramate & 0 & $3(8.3)$ & 0.24 \\
Poly-therapy & $4(13.7)$ & $9(25)$ & 0.35 \\
Others anti-epileptic drugs & $7(24.1)$ & $6(16)$ & 0.45 \\
\hline
\end{tabular}

more than one type of headache, like inter-ictal/preictal which was present in 12 patients (18.4\%), and one patient $(1.5 \%)$ had interictal/post-ictal, another one also $(1.5 \%) \mathrm{had}$ interictal/ ictal / post-ictal, and one patient $(1.5 \%)$ had ictal/pre-ictal headache.

There was no significant difference in age, gender, type of epilepsy syndrome, and the antiepileptic used in patients who had headaches compared to patients without headaches (Table 2).

In a subgroup analysis of patients with migraine compared with non-migraine headaches, there was no significant difference in age, gender, type of epilepsy syndrome, and antiepileptic drug used except for the valproic acid, which showed fewer migraine patients compared with non-migraine patients (Table 3).

Discussion. Our study shows that about $46 \%$ of patients with epilepsy had headache as a comorbidity compared to $2 \%$ in the control group. The link between headache and seizures is controversial, and the literature review on this topic for the pediatric age group is limited.

In a study by Kanemura et $\mathrm{al}^{4}$ that enrolled 98 pediatric epilepsy patients, 35\% of them had headache. Another study conducted by Yamane et $\mathrm{al}^{5}$ enrolled 50 pediatric epilepsy patients, and headache was reported by $46 \%$ of them, while Papavasiliou et $\mathrm{al}^{6}$ found that $11.4 \%$ among 70 pediatric epilepsy patients reported headache. Published studies in adult epileptic patients up to 2015 showed that the prevalence of migraine headache ranged from $6.6 \%$ to $32.9 \%$, and the minimum age included in those studies was 10 years. ${ }^{7,12,18-32}$ The most recent studies were published by Wang et $\mathrm{a}^{32}$ who interviewed 1109 adult patients aged 18 years or older, and they found that headache occurred in $12.5 \%$ of the patients.In 2015, Mainieri ${ }^{33}$ reported that $53.9 \%$ of epilepsy patients had headache.

In cohorts of migraine patients, epilepsy was reported infrequently. In a cohort of 172 headache patients, $1.7 \%$ had unprovoked seizures and 3 of 84 (2.3\%) had coexisting migraine and epilepsy. ${ }^{6}$ In another study that was conducted at a pediatric headache center and that enrolled 1,795 patients, $56(3.1 \%)$ patients also had epilepsy. Among these epileptic patients, $46 / 56$ (82\%) had migraine headache. ${ }^{34}$

The pathophysiology behind the relationship between headache and epilepsy was reviewed in 2008, particularly the migraine type. ${ }^{34}$ It is postulated that migraine attacks, similar to epileptic seizures, may be triggered by excessive neocortical cellular excitability. In migraine, this leads to cortical spreading depression and aura followed by additional recruitment of the trigeminal nucleus, resulting in central sensitization and pain. However, in epilepsy, neuronal overactivity can cause further neuronal recruitment and lead to firing in a rhythmic manner that constitutes an epileptic seizure. Migraine aura and headache may act as a trigger for epilepsy. ${ }^{35}$ Additionally, some forms of 
epilepsy and migraine are known to be channelopathies, which result from mutations in the same genes that can cause migraine, epilepsy, or both. This is similar to familial hemiplegic migraine syndromes where different mutations are found and can produce epilepsy, migraine, or both, and this can explain why some antiepileptic drugs, including valproate and topiramate, are effective in both conditions. ${ }^{34}$

For the timing of headache related to seizures, Yamane et $\mathrm{al}^{5}$ showed that about $60 \%$ of headaches were inter-ictal and the rest were pre- or post-ictal. In another group of pediatric and adult patients, $71 \%$ had inter-ictal headaches. ${ }^{36}$ These findings are similar to our study, where most headaches occurred in the inter-ictal period and affected $84 \%$ of our patients. However, Kanemura et $\mathrm{al}^{4}$ indicated that there was a higher prevalence in the post-ictal period in 28/34 (82\%) of their patients, which is not consistent with our, and others', results.In a group of adult patients, post-ictal headache was the most common type, especially in patients taking polytherapy; these patients have a higher seizure frequency, suggesting that a severe epilepsy phenotype and seizures can act as a trigger for headache attacks. ${ }^{28,32,30}$

The migraine type was also the most frequent type of headache seen in $44.6 \%$ of our patients, while tensiontype headache was present in $18.4 \%$ and unclassified headache was present in $36.9 \%$ of patients. Similar findings were reported in other studies such as Ottman et $\mathrm{al}^{7}$ who enrolled 1948 adult patients with epilepsy and demonstrated a two-fold higher risk for migraine in patients with epilepsy compared to their first-degree relatives without epilepsy. They also showed a two-fold higher risk of migraine compared to controls (24\% vs. $12 \%)$. In Yamane et $\mathrm{al}^{5}$ migraine was present in $43.5 \%$ of the patients and $17.4 \%$ had tension-type headache, while in $39.1 \%$ of the patients, the type of headache could not be established. Others reported migraine in more specific epileptic syndromes, such as benign rolandic epilepsy and benign occipital epilepsy of childhood, ${ }^{1,8,9}$ others found it more common in benign epilepsy with centrotemporal spikes and juvenile myoclonic epilepsy. ${ }^{11}$

In our study, migraine was observed less frequently in patients who took valproate compared to patients who received other treatments, indicting a potential preventative effect for migraine in epilepsy patients who are treated with this drug compared to other drugs. However, further studies are required to confirm this hypothesis. Although valproic acid was shown to be effective in adults, ${ }^{37}$ there are few controlled trials in children. A previous placebo-controlled trial showed the efficacy of topiramate for prevention of migraine in children, ${ }^{38}$ but a recent study comparing topiramate, amitriptyline, and placebo found no difference compared to placebo. ${ }^{39}$ In our study, topiramate was administered in epileptic patients with or without headache, and there was no statistically significant difference between the groups, which is in agreement with the findings of Powers et al. ${ }^{39}$

We also found that headache and epilepsy started in the same year in 28 patients $(43 \%)$, but headache preceded epilepsy in 19 patients (29\%) and epilepsy started before headache in 17 patients (26\%). This indicates that in most patients, the headache started in the same year with epilepsy. Our findings are comparable to the findings of Toldo et $\mathrm{al}^{34}$ where in $44 \%$ of patients, epilepsy started earlier than headache with $28.6 \%$ starting in the same year, while headache started before seizures in $27.4 \%$ of the patients. Yamane et al. showed that the headache usually starts in the same year or after an epilepsy diagnosis. ${ }^{5}$

The main limitation of our study is the recall bias of our patients and the cross-sectional nature. However, it has a relatively large sample size compared to other, similar studies and it is the only study in Saudi patients. A larger sample size with a prospective cohort study is required to better address the questions related to comorbidity of headache in pediatric epilepsy patients.

In conclusion, headache, predominantly migraine, is a common problem in pediatric epileptic patients, that can be secondary to similarity in their pathophysiology. A careful history related to headaches is recommended in those patients to improve their care and quality of life. Choosing valproic acid when possible can also be of importance in preventing migraine in these patients.

Acknowledgment. We would like to thanks American manuscript editing for English language editing. Also, we would like to thanks the radiologists in King Abdullah Children Specialist Hospital, King Abdul-Aziz Medical City, Riyadh, Saudi Arabia, for reviewing the images of the patients.

\section{References}

1. Andermann F. Migraine-epilepsy relationships. Epilepsy Research 1987; 1: 213-226.

2. Fisher RS, van Emde Boas W, Blume W, Elger C, Genton P, Lee $P$, et al. Epileptic seizures and epilepsy: definitions proposed by the International League Against Epilepsy (ILAE) and the International Bureau for Epilepsy (IBE). Epilepsia 2005; 46: 470-472.

3. Haut SR, Bigal ME, Lipton RB. Chronic disorders with episodic manifestations: focus on epilepsy and migraine. Lancet Neurol 2006; 5: 148-157.

4. Kanemura H, Sano F, Ishii S, Ohyama T, Sugita K, Aihara M. Characteristics of headache in children with epilepsy. Seizure 2013; 22: 647-650. 
5. Yamane LE, Montenegro MA, Guerreiro MM. Comorbidity headache and epilepsy in childhood. Neuropediatrics 2004; 35: 99-102.

6. Papavasiliou AS, Bregianni M, Nikaina I, Kotsalis C, Paraskevoulakos E, Bazigou H. Pediatric headache and epilepsy comorbidity in the pragmatic clinical setting. Neuropediatrics 2016; 47: 107-111.

7. Ottman R, Lipton RB. Comorbidity of migraine and epilepsy. Neurology 1994; 44: 2105-2110.

8. Andermann F, Zifkin B. The benign occipital epilepsies of childhood: an overview of the idiopathic syndromes and of the relationship to migraine. Epilepsia 1998; 39: S9-S23.

9. Panayiotopoulos CP. Elementary visual hallucinations, blindness, and headache in idiopathic occipital epilepsy: differentiation from migraine. J Neurol Neurosurg Psychiatry 1999; 66: 536-540.

10. Thomsen LL, Kirchmann M, Bjornsson A, Stefansson H, Jensen RM, Fasquel AC, et al. The genetic spectrum of a populationbased sample of familial hemiplegic migraine. Brain 2007; 130: 346-356.

11. Kelley SA, Hartman AL, Kossoff EH. Comorbidity of migraine in children presenting with epilepsy to a tertiary care center. Neurology 2012; 79: 468-473.

12. Schon F, Blau JN. Post-epileptic headache and migraine. $J$ Neurol Neurosurg Psychiatry 1987; 50: 1148-1152.

13. Parisi P. Why is migraine rarely, and not usually, the sole ictal epileptic manifestation? Seizure 2009; 18: 309-312.

14. Sasmaz T, Bugdayci R, Ozge A, Karakelle A, Kurt O, Kaleagasi H. Are parents aware of their schoolchildren's headaches? Eur J Public Health. 2004; 14: 366-368.

15. Headache Classification Subcommittee of the International Headache Society. The International Classification of Headache Disorders: 2nd edition. Cephalalgia 2004; 24: 9-160.

16. Proposal for revised classification of epilepsies and epileptic syndromes. Commission on Classification and Terminology of the International League Against Epilepsy. Epilepsia 1989; 30: 389-399.

17. Classification and diagnostic criteria for headache disorders, cranial neuralgias and facial pain. Headache Classification Committee of the International Headache Society. Cephalalgia 1988; 8: 1-96.

18. Ito M, Schachter SC. Frequency and characteristics of interictal headaches in patients with epilepsy. Journal of Epilepsy 1996; 9: 83-86.

19. Ito M, Nakamura F, Honma H, Takeda Y, Kobayashi R, Miyamoto T, et al. A comparison of post-ictal headache between patients with occipital lobe epilepsy and temporal lobe epilepsy. Seizure 1999; 8: 343-346.

20. Velioğlu SK, Ozmenoğlu M. Migraine-related seizures in an epileptic population. Cephalalgia 1999; 19: 797-801.

21. Leniger T, Isbruch K, von den Driesch S, Diener HC, Hufnagel A. Seizure-associated headache in epilepsy. Epilepsia 2001; 42: 1176-1179.
22. Karaali-Savrun F, Goksan B, Yeni SN, Ertan S, Uzun N. Seizure-related headache in patients with epilepsy. Seizure 2002; 11: 67-69.

23. Forderreuther S, Henkel A, Noachtar S, Straube A. Headache associated with epileptic seizures: epidemiology and clinical characteristics. Headache 2002; 42: 649-655.

24. Ito M, Adachi N, Nakamura F, Koyama T, Okamura T, Kato $\mathrm{M}$, et al. Characteristics of postictal headache in patients with partial epilepsy. Cephalalgia 2004; 24: 23-28.

25. Syvertsen M, Helde G, Stovner LJ, Brodtkorb E. Headaches add to the burden of epilepsy. J Headache Pain 2007; 8: 224-230.

26. Kwan P, Man CB, Leung H, Yu E, Wong KS. Headache in patients with epilepsy: a prospective incidence study. Epilepsia 2008; 49: 1099-1102.

27. HELP Study Group. Multi-center study on migraine and seizure-related headache in patients with epilepsy. Yonsei Med J 2010; 51: 219-224.

28. Tonini MC, Giordano L, Atzeni L, Bogliun G, Perri G, Saracco MG, et al. Primary headache and epilepsy: a multicenter crosssectional study. Epilepsy Behav 2012; 23: 342-347.

29. Duchaczek B, Ghaeni L, Matzen J, Holtkamp M. Interictal and periictal headache in patients with epilepsy. Eur J Neurol 2013; 20: 1360-1366.

30. Winawer MR, Connors R, Investigators EPGP. Evidence for a shared genetic susceptibility to migraine and epilepsy. Epilepsia 2013; 54: 288-295.

31. Gameleira FT, Ataíde L Jr, Raposo MC. Relations between epileptic seizures and headaches. Seizure 2013; 22: 622-626.

32. Wang XQ, Lang SY, He MW, Zhang X, Zhu F, Dai W, et al. High prevalence of headaches in patients with epilepsy. $J$ Headache Pain 2014; 15: 70.

33. Mainieri G, Cevoli S, Giannini G, Zummo L, Leta C, Broli M, et al. Headache in epilepsy: prevalence and clinical features. $J$ Headache Pain 2015; 16: 556.

34. Toldo I, Perissinotto E, Menegazzo F, Boniver C, Sartori S, Salviati L, et al. Comorbidity between headache and epilepsy in a pediatric headache center. J Headache Pain 2010; 11: 235-240.

35. Nye BL, Thadani VM. Migraine and epilepsy: review of the literature. Headache 2015; 55: 359-380.

36. Hofstra WA, Hageman G, de Weerd AW. Periictal and interictal headache including migraine in Dutch patients with epilepsy: a cross-sectional study. Epilepsy Behav 2015; 44: 155-158.

37. Linde M, Mulleners WM, Chronicle EP, McCrory DC. Valproate (valproic acid or sodium valproate or a combination of the two) for the prophylaxis of episodic migraine in adults. Cochrane Database Syst Rev 2013; 6: CD010611.

38. Fallah R, Divanizadeh MS, Karimi M, Mirouliaei M, Shamszadeh A. Topiramate and propranolol for prophylaxis of migraine. Indian J Pediatr 2013; 80: 920-924.

39. Powers SW, Coffey CS, Chamberlin LA, Ecklund DJ, Klingner EA, Yankey JW, et al. Trial of Amitriptyline, Topiramate, and Placebo for Pediatric Migraine. N Engl J Med 2017; 376: 115-124. 